\title{
Etude hydroacoustique d'une vanne papillon en régime cavitant
}

\author{
Hydroacoustic study of a butterfly valve in cavitating flow
}

\author{
par A. Boyer, J.-F. Lauro \\ EDF/DER/Département Machines
}

The CIRCUS code (EDF/DER) allows to determine the pipework network behaviour in case of a flow submitted to hydroacoustic excitations, generally due to some network special devices such as pumps or valves. CIRCUS uses a data base which characterizes each network component through its tranfer matrix and its hydroacoustic source. The butterfly valve in cavitating flow is a really complex component, particularly because waves propagation speed is not constant in the cavitating zone. Numerous tests have been done on the EPOCA loop in Chatou where parameters such as valve opening, flow rate et cavitation rate were examined. Waves propagation speed in the cavitating zone as well as cavitation length were charaterized. Transfer Matrix was determined and the hydroacoustic source was localized downstream of the valve and estimated.

\section{LE CONTEXTE ET LES OBJECTIFS}

Les phénomènes hydroacoustiques qui perturbent les circuits hydrauliques ont pour origine certains composants, comme les pompes, les organes de réglage de débit,... En particulier, les organes cavitants (et notamment les vannes papillon) génèrent des perturbations hydroacoustiques très importantes. Leur influence en terme de génération et de transmission d'ondes sur le comportement vibro-acoustique de réseaux de tuyauteries doit être prise en compte.

Afin de prédire le comportement acoustique et vibratoire des circuits hydrauliques sous écoulement, la DER a développé le code CIRCUS. Mais l'utilisation de ce code nécessite la connaissance des caractéristiques hydroacoustiques des composants des circuits. C'est pour alimenter la base de données de CIRCUS que le Département machines a entrepris l'étude des vannes en régime cavitant.

Le but de cette étude est de déterminer un modèle hydroacoustique de type sources/matrice de transfert, représentant le comportement d'une vanne Papillon en régime cavitant en s'appuyant sur une base de données expérimentale issue d'essais réalisés sur la boucle EPOCA à différents régimes de fonctionnement pour une vanne Papillon $\varnothing 200$.

Une première campagne d'essais, réalisée début 1995, a permis de mieux comprendre les phénomènes mis en jeu:
- La cavitation se décompose en deux phénomènes, une zone de structure cavitante et un jet de microbulles.

- Il y a une bonne corrélation spatiale et fréquentielle entre l'analyse des signaux de fluctuation de pression et la visualisation pour ce qui concerne la zone à grosses structures.

- Par contre, la transition hydroacoustique entre un régime non cavitant et un régime faiblement cavitant ne suit pas l'apparition visuelle de la cavitation.

- En dehors de la zone cavitante, l'hypothèse d'une propagation en ondes planes est démontrée.

- La mise en œuvre aisée de l'excitatrice de débit permet de générer des perturbations hydroacoustiques très supérieures à celles générées par la vanne.

Une deuxième campagne d'essais a été réalisée fin 1996. Les principaux objectifs étaient :

- réunir une base de données expérimentale de bonne qualité,

- vérifier qu'il est faisable de décrire le comportement hydroacoustique d'une vanne Papillon en régime cavitant sous la forme source/matrice de transfert,

- déterminer les paramètres d'un ou de plusieurs modèles et valider ces modèles à l'aide du code CIRCUS.

Les moyens et les méthodes utilisées, les modèles proposés, les principaux résultats et les perspectives sont précisés dans les chapitres suivants. 


\section{DES DIFFÉRENTS MODÈLES DE VANNE PROPOSÉS et TESTÉS}

\subsection{Les modèles hydroacoustiques de type sources/matrice de transfert}

Pour décrire le comportement hydroacoustique d'une partie de circuit hydraulique, on utilise une formulation matricielle ; c'est d'ailleurs sur ce principe que fonctionne le code CIRCUS. La relation entre les vecteurs d'état de 2 points successifs d'un circuit s'écrit donc :

$$
\left[\begin{array}{l}
p 2 \\
q 2
\end{array}\right]=[M] \times\left[\begin{array}{l}
p 1 \\
q 1
\end{array}\right] \text { avec }[M]=\left[\begin{array}{cc}
\cos \frac{\omega L}{c} & -i \frac{\rho c}{S} \sin \frac{\omega L}{c} \\
-i \frac{S}{\rho c} \sin \frac{\omega L}{c} & \cos \frac{\omega L}{c}
\end{array}\right]
$$

dans le cas où les 2 points sont séparés par une tuyauterie droite de section $S$ et de longueur $L$, en considérant un modèle de propagation en ondes planes et une vitesse d'écoulement négligeable par rapport à la vitesse des ondes $c$.

Il est possible de décrire par une telle formulation - en utilisant au besoin une succession de matrices - toutes les parties "passives " d'un circuit hydraulique. Dans le cas où le circuit comporte un composant « actif », i.e. un composant qui génère des perturbations hydroacoustiques, il faut ajouter à la formulation un vecteur source. L'équation devient :

$$
\left[\frac{p 2}{q 2}\right]=[M] \times\left[\frac{p 1}{p 2}\right]+\left[\frac{\Delta p s}{\Delta q s}\right]
$$

A partir de ces deux éléments (matrice de transfert et vecteur source) il est possible de décrire complètement le comportement hydroacoustique d'un circuit quelconque, à condition, bien entendu, de disposer d'une représentation matricielle de chaque composant du circuit.

Dans le chapitre suivant, nous allons présenter plusieurs modèles utilisables pour la représentation du comportement hydroacoustique d'une vanne Papillon.

\subsection{Les modèles hydroacoustiques de vanne en régime cavitant}

\subsubsection{Le modèle "Standard"}

Un modèle hydroacoustique dit «à 3 zones homogènes " avait déjà été testé ; nous l'appellerons " modèle standard ».

Dans ce modèle, la vanne est représentée par :

- une matrice de transfert $[M]$ ponctuelle située en son centre, - un vecteur source $[\Delta p s, \Delta q s]$ à l'aval immédiat,

- une matrice de transfert $[M c]$ de longueur $L c$ et où la propagation se fait à une vitesse $C c$ représentant la zone où se développe la cavitation.

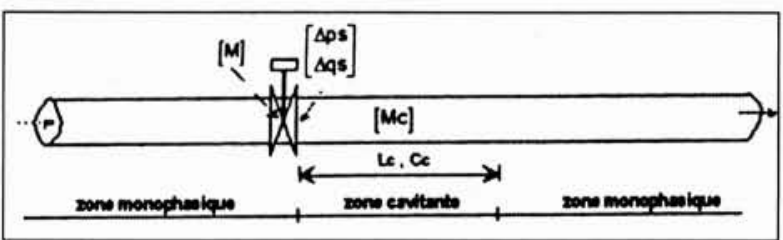

$$
\left[\begin{array}{l}
p 2 \\
q 2
\end{array}\right]=[M c] \times[M] \times\left[\begin{array}{l}
p 1 \\
q 1
\end{array}\right]+[M c] \times\left[\begin{array}{c}
\Delta p s \\
\Delta q s
\end{array}\right]
$$

1. Modèle de vanne cavitante à 3 zones homogènes, dit « modèle standard ".
Deux variantes développées à partir du modèle « standard» ont été testées :

- un modèle à deux sources de débit et une matrice de transfert non ponctuelle ;

- un modèle dit «à 5 zones", i.e. une matrice ponctuelle caractéristique de la singularité, une source à l'aval suivie de 4 matrices de transmission à différentes vitesses.

\subsubsection{Modèle à deux sources de débit}

Dans le modèle à 2 sources de débit, la vanne est représentée par:

- une source amont $[0, q s a]$ située au centre de la vanne,

- une matrice de transfert $[M v]$ de longueur $L v$ qui représente la zone située immédiatement à l'aval de la vanne contenant les structures de cavitation,

- une source aval $[0, q s r]$ située approximativement à la fin de la zone des grosses structures,

- une matrice de transfert $[M c]$ de longueur $L c$ et où la propagation se ferait à une vitesse $C c$; cette partie correspond au jet de microbulles.

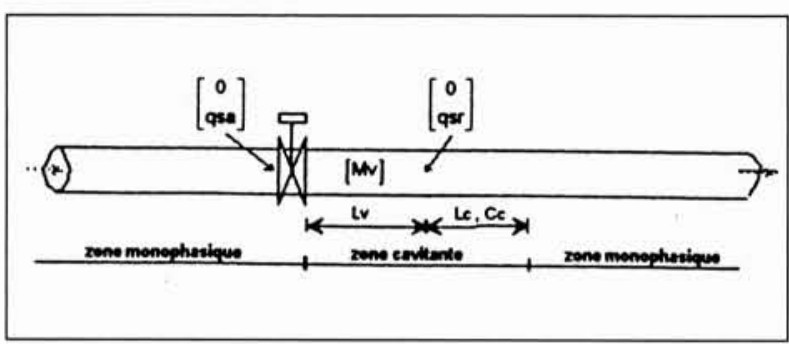

$\left[\begin{array}{l}p 2 \\ q 2\end{array}\right]=[M c] \times[M v] \times\left(\left[\begin{array}{c}p 1 \\ q 1\end{array}\right]+\left[\begin{array}{c}0 \\ q s a\end{array}\right]\right)+[M c] \times\left[\begin{array}{c}0 \\ q s r\end{array}\right]$

\section{Modèle de vanne cavitante à deux sources de débit.}

La formulation correspond très bien aux concepts mis en œuvre dans le code CIRCUS où les chargements extérieurs sont les débits et où les pressions sont les conséquences de ces chargements.

Il n'est pas nécessaire de connaître a priori la vitesse des ondes dans la partie représentée par la matrice $[M v]$, les 4 termes de cette matrice étant déterminés expérimentalement.

Ce modèle correspond moins bien au phénomène physique tel qu'il a été observé dans une vanne cavitante, mais il est toujours possible de revenir d'une formulation à une source $\left[\Delta p s^{\prime}, \Delta q s^{\prime}\right]$ par une transposition.

\subsubsection{Modèle à cinq zones}

Dans le modèle à 5 zones, la vanne est représentée par : - une matrice de transfert $[M]$ ponctuelle située au centre de la vanne,

- une matrice de transfert $[M 1]$ de longueur $L 1$ où la vitesse de propagation est $C 1$ représentant la zone de grosses structures,

- une matrice de transfert $[M 2]$ de transition, de longueur $L 2$ et de vitesse $C 2$,

- une source aval $[\Delta p s, \Delta q s]$ située approximativement à la fin de la zone des grosses structures,

- une matrice de transfert $[M 3]$ de longueur $L 3$ et de vitesse $C 3$ qui correspondrait à la zone des microbulles, - une matrice de transfert [M4] de transition, de longueur $L 4$ et de vitesse $C 4$. 
C'est ce modèle relativement complexe qui représente le plus fidèlement les phénomènes physiques tels qu'ils ont été observés lors des visualisations effectuées à travers une manchette transparente implantée à l'aval de la vanne.

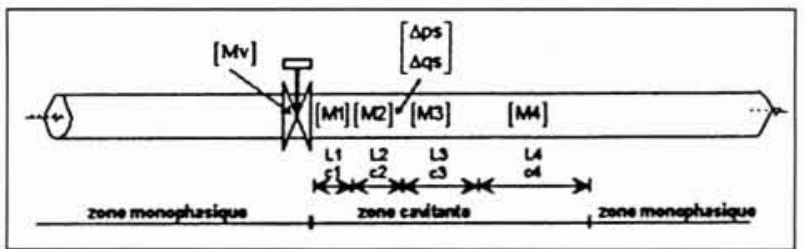

3. Modèle de vanne cavitante à 5 zones.

$$
\left[\begin{array}{l}
p 2 \\
q 2
\end{array}\right]=[M 4] \times[M 3] \times\left([M 2] \times[M 1] \times[M v] \times\left[\begin{array}{l}
p 1 \\
q 1
\end{array}\right]+\left[\begin{array}{c}
\Delta p s \\
\Delta q s
\end{array}\right]\right)
$$

\section{III — LES MOYENS ET LES MÉTHODES MIS EN OEUVRE}

\section{- 3.1 Les moyens}

La veine d'essais implantée sur la boucle EPOCA est présentée sur la figure 4 ; elle est constituée d'une conduite rectiligne $\varnothing 200$ de $9,6 \mathrm{~m}$ environ, reliée au circuit principal par 2 compensateurs élastomères (DILATOFLEX). La veine est équipée de 25 capteurs de pression fluctuante KISTLER. A chaque extrémité, il est possible de connecter une excitatrice de débit à vanne rotative, elle-même munie d'un capteur de pression fluctuante.

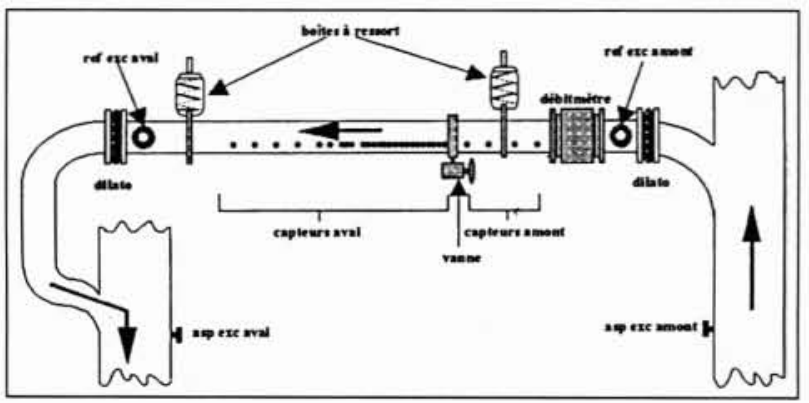

4. Schéma de la veine d'essai.

Les signaux sont numérisés par l'intermédiaire d'un frontal SCADAS (DIFA) piloté par le logiciel FMON (LMS), transformés dans le domaine fréquentiel, puis transférés vers le logiciel LADY (INTESPACE/DER) où sont implantés les traitements d'intensimétrie hydroacoustique et les modèles de composants que nous cherchons à identifier.

\section{- 3.2 Les méthodes de détermination de la matrice de transfert et des sources}

Afin de simplifier, nous allons expliquer la démarche pour le modèle standard. La démarche est identique pour les 2 autres modèles : détermination de la matrice de transfert puis dans un second temps de la source associée.

La vitesse des ondes propagatives est préalablement déterminée pour chaque triplet de capteurs équidistants par intensimétrie.

\subsubsection{La détermination de la matrice de transfert}

Pour déterminer la matrice de transfert, on réalise 2 essais (a et b) avec une source externe située à l'extérieur de la zone instrumentée, successivement à l'amont (essai a) puis à l'aval (essai b). On vérifie que les sources externes sont nettement supérieures à la source générée par la vanne et on obtient les deux équations suivantes:

$$
\left[\begin{array}{l}
p 2 a \\
q 2 a
\end{array}\right]=[M c] \times[M] \times\left[\begin{array}{l}
p 1 a \\
q 1 a
\end{array}\right] \quad\left[\begin{array}{l}
p 2 b \\
q 2 b
\end{array}\right]=[M c] \times[M] \times\left[\begin{array}{l}
p 1 b \\
q 1 b
\end{array}\right]
$$

La matrice $[M c]$ étant connue $\left({ }^{1}\right)$, on dispose de 4 équations pour déterminer les 4 termes inconnus de la matrice [M].

\subsubsection{L'identification de la source " associée "}

Un troisième essai $(e)$ réalisé sans source externe permet, ensuite, de déterminer les sources $([M c]$ et $[M]$ étant connues) à partir de l'équation suivante :

$$
\left[\begin{array}{l}
p 2 e \\
q 2 e
\end{array}\right]=[M c] \times[M] \times\left[\begin{array}{l}
p 1 e \\
q 1 e
\end{array}\right]+[M c] \times\left[\begin{array}{c}
\Delta p s \\
\Delta q s
\end{array}\right]
$$

En pratique, les 3 modèles ont été implantés dans un module privé de LADY qui se charge d'effectuer les calculs nécessaires à partir des données expérimentales.

\subsection{Les essais réalisés}

Pendant la campagne d'essai, 42 régimes de fonctionnement ont été étudiés en faisant varier trois grandeurs : l'ouverture de la vanne, le débit et le nombre de THOMA $(\sigma)$ dans les limites permises par la boucle d'essais.

\begin{tabular}{|c|c|c|c|c|c|c|}
\hline & $Q\left(\mathrm{~m}^{3} / \mathrm{h}\right)$ & 170 & 280 & 395 & 510 & 620 \\
\hline & $V(\mathrm{~m} / \mathrm{s})$ & 1,5 & 2,5 & 3,5 & 4,5 & 5,5 \\
\hline \multirow{5}{*}{$\begin{array}{c}\text { Ouverture } \\
n^{*} 1 \\
63 \% \\
\left(33^{\circ}\right)\end{array}$} & $\sigma 5=2,5$ & & & A5 & B5 & C5 \\
\hline & $\sigma 6=3,0$ & & & $A 6$ & B6 & C6 \\
\hline & $\sigma 7=3,6$ & & & $A 7$ & $B 7$ & C7 \\
\hline & $\sigma 8=5,4$ & & & $A 8$ & B8 & C8 \\
\hline & $\sigma 9=8,2$ & & & $A 9$ & & \\
\hline \multirow{6}{*}{$\begin{array}{c}\text { Ouverture } \\
n^{\circ} 2 \\
51 \% \\
\left(44^{\circ}\right)\end{array}$} & $\sigma 3=1,6$ & & & E3 & F3 & \\
\hline & $\sigma 4=2,0$ & & & E4 & F4 & \\
\hline & $\sigma 5=2,5$ & & D5 & E5 & F5 & \\
\hline & $\sigma 6=3,0$ & & D6 & E6 & F6 & \\
\hline & $\sigma 7=3,6$ & & D7 & E7 & & \\
\hline & $\sigma 8=5,4$ & & $D 8$ & E8 & & \\
\hline \multirow{7}{*}{$\begin{array}{c}\text { Ouverture } \\
\mathbf{n}^{\circ} \mathbf{3} \\
\mathbf{3 9} \% \\
\left(5^{\circ}\right)\end{array}$} & $\sigma 1=0,7$ & & & 11 & & \\
\hline & $\sigma 2=1,0$ & & $\mathrm{H} 2$ & 12 & & \\
\hline & $\sigma 3=1,6$ & & H3 & 13 & & \\
\hline & $\sigma 4=2,0$ & G4 & H4 & 14 & & \\
\hline & $\sigma 5=2,5$ & G5 & H5 & 15 & & \\
\hline & $\sigma 6=3,0$ & G6 & H6 & & & \\
\hline & $\sigma 7=3,6$ & G7 & H7 & & & \\
\hline
\end{tabular}

(1) C'est la matrice de transfert d'un tuyau droit de longueur $L c$ et de vitesse Cc. 


\section{IV — LES RÉSULTATS}

Quelques exemples illustrent la démarche de l'analyse présentée au $\S 3$ et les modèles proposés au $\$ 2$.

\subsection{L'analyse des spectres de pression}

Des essais ont été effectués à une ouverture de la vanne de $51 \%$ et à une vitesse moyenne de $3,5 \mathrm{~m} / \mathrm{s}$. La boucle EPOCA a permis de faire varier le taux de cavitation $\sigma$ entre 1,6 et 5,4 .

L'analyse directe des spectres de fluctuation de pression (fig. 5) montre un brusque changement de leur allure à partir de $\sigma=3$. Au-dessus de cette valeur, le taux de cavitation n'intervient pas sur l'amplitude des fluctuations de pression. En dessous, l'allure des spectres se modifie régulièrement et les amplitudes augmentent nettement.

Lors de la première campagne d'essai, les photographies et les films rapides réalisés pour différents états de développement de la cavitation ont montré que pour les conditions de cet essai $(3,5 \mathrm{~m} / \mathrm{s}$ et $51 \%$ d'ouverture), la cavitation apparaît vers $\sigma=5$ environ, donc longtemps avant que son effet soit visible sur les fluctuations de pression (après $\sigma=3$ ). Ce résultat très important est confirmé.

\section{Remarque}

A partir de ce résultat, il faudra donc distinguer l'apparition de la cavitation du point de vue hydraulique (vers $\sigma=5$ pour cet essai par exemple) et l'apparition de la cavitation du point de vue hydroacoustique (vers $\sigma=3$ ). Dans cet article, lorsque nous parlerons d'un régime cavitant, nous nous placerons sur le plan hydroacoustique. L'observation des spectres de fluctuation de pression en fonction de la distance par rapport à la vanne (fig. 5) montre que le niveau maximum est mesuré à environ $30 \mathrm{~cm}$ à l'aval de la vanne. Cette position est la même quel que soit le degré de développement de la cavitation $\left(^{2}\right)$.

\subsection{La vitesse de propagation}

Un traitement des mesures par une méthode d'intensimétrie hydroacoustique permet de déterminer les valeurs de la célé- rité des ondes le long de la veine d'essai. L'analyse des résultats a montré qu'il faut considérer 3 zones :

- A l'amont de la vanne, la vitesse des ondes est constante et élevée. Sa valeur est pratiquement constante quelles que soient les conditions d'essai $C=1320 \mathrm{~m} / \mathrm{s}$, ce qui est très proche de la valeur théorique donnée pour une conduite équivalente par la formule d'ALLIEVI, $C=1335 \mathrm{~m} / \mathrm{s}$.

- A l'aval lointain, la vitesse retrouve une valeur pratiquement identique à celle de l'amont, sauf pour quelques essais qui présentent une cavitation très développée, où la vitesse chute pour atteindre - dans un cas $-700 \mathrm{~m} / \mathrm{s}\left({ }^{3}\right)$.

- A l'aval proche, la détermination de la vitesse des ondes reste délicate, essentiellement pour deux raisons :

- la connaissance fine de ce paramètre nécessite le rapprochement des capteurs, ce qui - paradoxalement - augmente la difficulté ( $1 / 2 \emptyset$ semble être la limite) ;

- la turbulence provoquée par la vanne masque les phénomènes acoustiques pris en compte par l'intensimétrie.

Mais, si la détermination de la vitesse des ondes reste imprécise dans cette zone, la valeur ne descend jamais audessous de $500 \mathrm{~m} / \mathrm{s}$. En effet, deux méthodes originales ont été testées avec succès au cours de cette campagne :

- La vitesse des ondes est identique avec et sans l'excitatrice hydroacoustique - toutes conditions fixées par ailleurs. Le niveau élevé produit par l'excitatrice permet de " masquer » la turbulence et améliore l'analyse intensimétrique.

- Une autre approche originale consiste à obtenir directement la vitesse par la mesure du temps mis par une onde pour parcourir une distance donnée. Dans notre cas, l'onde est un pic de pression émis par l'excitatrice et la distance est celle entre les capteurs. Cette méthode très simple s'est avérée très efficace, et si elle n'a pas permis, pour des raisons de matériel $\left({ }^{4}\right)$, de déterminer avec précision la vitesse en

(2) Elle est même plus marquée en régime non cavitant. Il semble que le maximum est plutôt dû à la turbulence.

(3) Ceci traduit probablement le fait que si le jet de microbulles n'arrive pas à se résorber, même après plusieurs mètres, les résorbeurs de la boucle EPOCA jouent parfaitement leur rôle, puisque la vitesse est toujours constante à l'amont. Les essais de visualisation avaient montré « qu'un jet de microbulles apparaît au centre la section en cavitation élevée et se continue au-delà de la section de visualisation sans qu'il soit possible de déterminer sa longueur .

(4) L'acquisition est limité à $20 \mathrm{kHz}$, soit une incertitude de l'ordre de $200 \mathrm{~m} / \mathrm{s}$ avec des capeurs distants de $10 \mathrm{~cm}$.

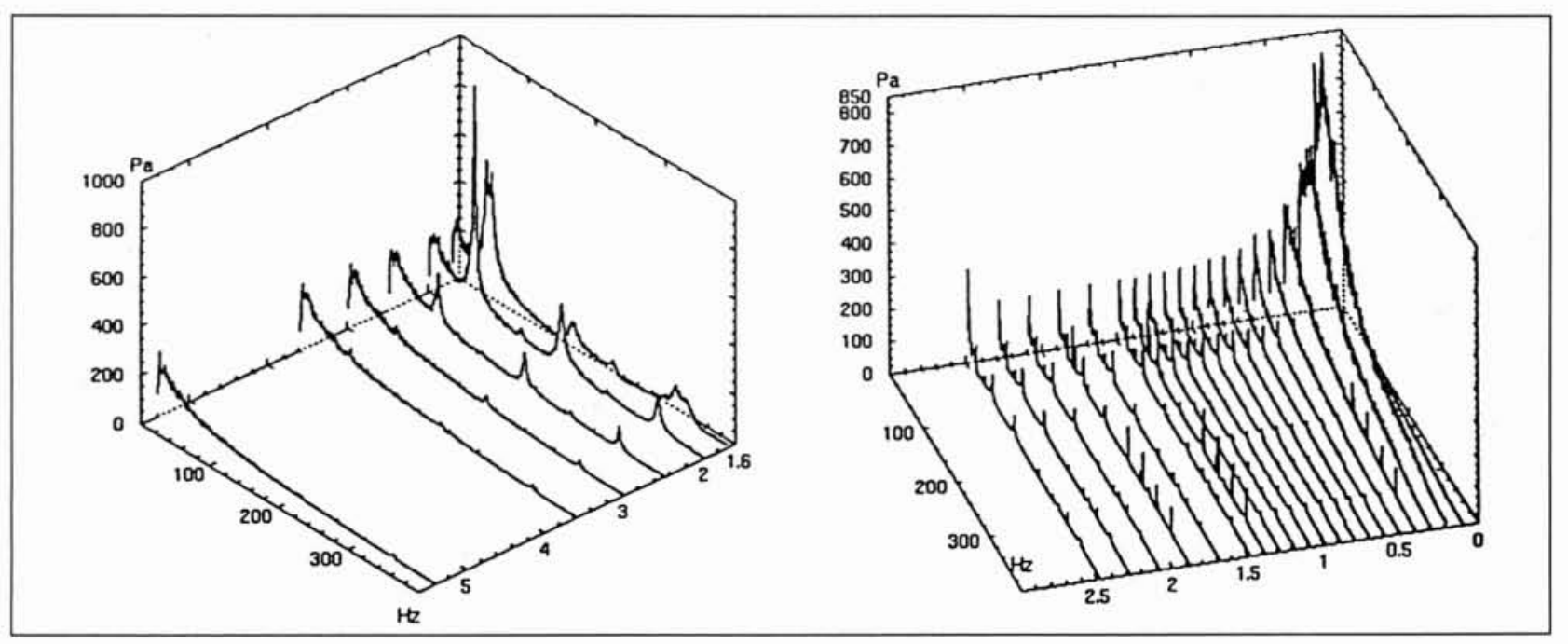

5. Spectres de pression en fonction de la cavitation (à gauche) et de la position à l'aval de la vanne (à droite). 
champ proche, elle a permis de confirmer les valeurs trouvées par ailleurs. Il est d'autre part possible d'obtenir une information sur le temps de transit dans la vanne en considérant 2 capteurs situés de part et d'autre, ce qu'aucune autre méthode ne permet.

A l'issue des essais, l'observation de l'ensemble des profils expérimentaux obtenus a permis de définir 3 types de profils de vitesse schématisés sur la figure 6:

- un profil normal qui correspond à la majorité des régimes étudiés (jusqu'à une cavitation déjà avancée),

- un profil " intermédiaire ", où la vitesse des ondes ne retrouve pas à l'aval la valeur atteinte à l'amont,

- un profil «très cavitant " qui correspond aux 2 points extrêmes.

\section{Conclusion}

Les deux méthodes originales de détermination de la célérité des ondes ont permis de montrer que la vitesse des ondes dans la zone cavitante est de l'ordre de $500 \mathrm{~m} / \mathrm{s}$.

Malgré d'excellentes mesures, la détermination de la célérité des ondes dans la zone cavitante est toujours délicate.
Nous considérerons donc les 3 profils «moyens " pour les modèles «standard " et à deux sources de débit.

Dans le cas du modèle à 5 zones, les profils expérimentaux seront directement pris en compte.

\subsection{Les matrices de transfert}

L'excitatrice hydroacoustique à vanne tournante développée par le Département Machines a été mise en œuvre sans difficulté sur la boucle EPOCA. Elle a permis de générer des fluctuations de débit qui représentent des niveaux environ 5 à 20 fois supérieurs aux perturbations engendrées par la vanne Papillon. Des mesures ont été réalisées pour un même régime avec l'excitatrice à l'amont, puis avec l'excitatrice à l'aval de la veine d'essais. Les 4 termes de la matrice de transfert ont été identifiés pour les trois modèles $\left({ }^{5}\right)$.

(5) Pour des raisons de sécurité (risque de résonance de la boucle EPOCA), la fréquence minimale d'excitation a été fixée $16 \mathrm{~Hz}$ environ. Il ne faut done pas prendre en compte les valeurs expérimentales en dessous de cette fréquence.

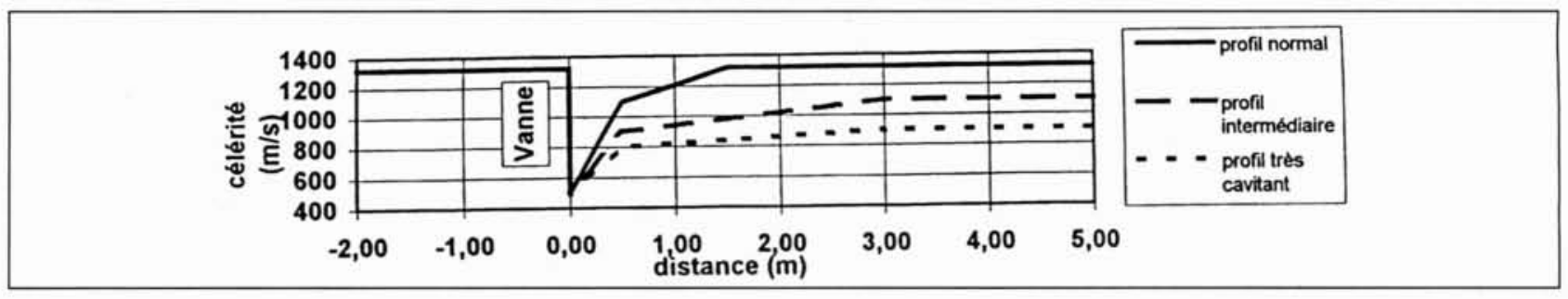

6. Schéma des 3 types de profils de vitesse.

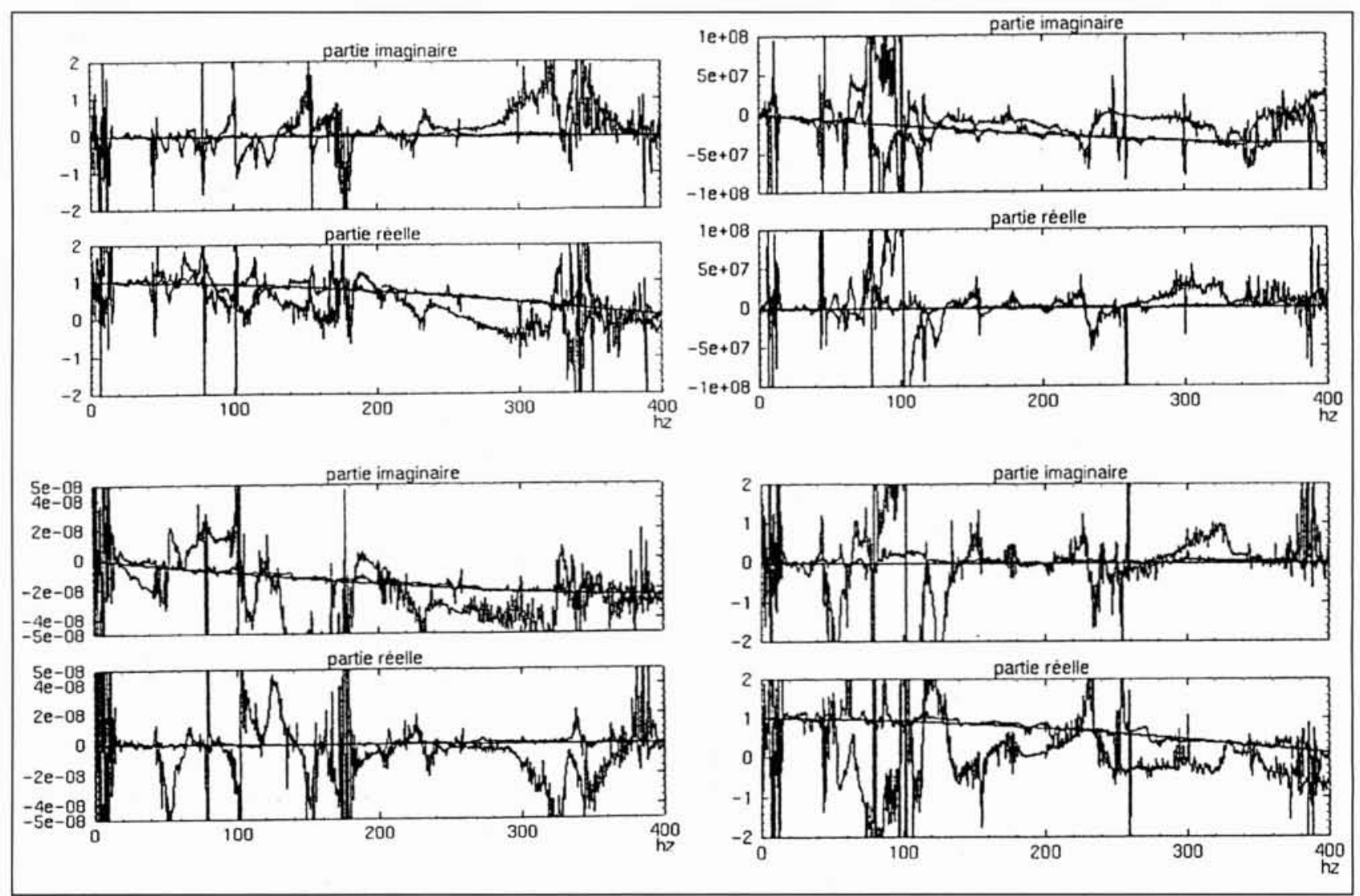

7. Spectres des 4 termes de la matrice de transfert pour le modèle à 2 sources de débit pour un essai peu cavitant $(\sigma=5,4)$ et pour un essai cavitant $(\sigma=2)$. 
Pour les 2 modèles à matrice ponctuelle (modèles standard et à 5 zones), la matrice obtenue est très proche de la matrice identité en normant par rapport à l'impédance caractéristique du circuit $Z_{0}\left({ }^{6}\right)$.

Pour le modèle à matrice répartie (à 2 sources), la matrice est très proche de la matrice d'un tuyau droit de longueur équivalente et de vitesse de propagation $C c$.

Dans tous les cas, la similitude est grande entre les matrices obtenues en régime cavitant et en régime peu cavitant. En régime cavitant, la source externe fournie par l'excitatrice est seulement 5 fois supérieure aux perturbations générées par la vanne, ce qui explique la difficulté de détermination et l'allure irrégulière des spectres de la matrice.

\section{Conclusion}

Les résultats montrent que les ondes sont peu affectées lors de leur passage à travers la vanne $\left({ }^{7}\right)$, quel que soit le régime de fonctionnement ; à part pour les 2 régimes extrêmes, pour lesquels la matrice est relativement différente.

\subsection{Les sources}

La connaissance de la matrice de transfert et la réalisation d'un essai supplémentaire sans excitation extérieure permet d'identifier les sources associées pour les 3 modèles proposés.

\subsubsection{Remarque}

Nous avons pris comme origine de phase la source de débit $(\Delta q s)$ pour les modèles "standard " et à 5 zones ; et la source de débit aval $(q s r)$ pour le modèle à deux sources de débit.

Pour le modèle standard, la détermination paraît correcte. L'amplitude des sources de pression et de débit est indépendante du taux de cavitation en basse fréquence. Pour les fréquences plus élevées, l'amplitude augmente fortement avec le taux de cavitation à partir de $\sigma=3$.

(6) $\mathrm{Z}_{0}$ est l'impédance caractéristique de la conduite. Pour un tronçon donné, $\mathrm{Z} 0=(\rho . c) / \mathrm{S}$ avec $\rho=$ masse volumique, $\mathrm{c}=$ célérité des ondes, $\mathrm{S}=$ section de la conduite.

(7) Mise à part, bien entendu, la modification de la vitesse de propagation à l'aval.
Le déphasage entre les 2 sources est globalement de $\pi / 2$, ce qui correspond à la théorie (impédance imaginaire).

Pour le modèle à 5 zones, les résultats sont du même ordre de grandeur. Cependant la qualité de détermination paraît moins bonne ; en effet, il subsiste une forte participation modale sur les spectres des sources, particulièrement pour la source de pression en régime cavitant.

Pour le modèle à deux sources de débit, la détermination est excellente, particulièrement pour les régimes peu cavitants. Pour les régimes cavitants, il subsiste une légère participation modale à l'amont ( $q s a)$ mais beaucoup moins que dans le cas précédant. Plusieurs remarques peuvent être faites à propos des spectres de ces sources:

- les deux sources sont identiques à l'amont et à l'aval pour les régimes non cavitants ; pour les régimes cavitants, la différence est plus importante sur les modes, mais c'est là que la détermination est la moins bonne,

- pour tous les régimes, l'amplitude est identique jusqu'à $80 \mathrm{~Hz}$ environ ; après cette valeur, l'amplitude augmente avec la cavitation,

- les deux sources sont en opposition de phase en régime non cavitant, elles sont en opposition de phase en basse fréquence et en phase en haute fréquence en régime cavitant ; le changement de phase dépend de la cavitation.

\subsubsection{Conclusion}

La «meilleure allure " des paramètres du modèle à 2 sources de débit n'est pas un indice suffisant pour choisir entre les trois modèles. Dans le $\S 5$, nous allons montrer la démarche de validation des modèles.

\section{V — La validation des modèles}

\subsection{Le principe}

Il y a plusieurs façons de valider des modèles hydroacoustiques de singularité obtenus expérimentalement.

- Le logiciel LADY permet, par intensimétrie, de calculer le vecteur d'état $(p 2, q 2)$ en un point quelconque situé à l'aval à partir d'un vecteur amont $(p 1, q 1)$, de la matrice de transfert $[M]$ et de la source $(\Delta p s, \Delta q s)$; on compare ensuite avec la mesure réalisée à ce point. Cette validation est simple et rapide, mais très peu efficace ; en effet il s'agit de recalculer une grandeur d'entrée qui avait servi à déterminer les incon-

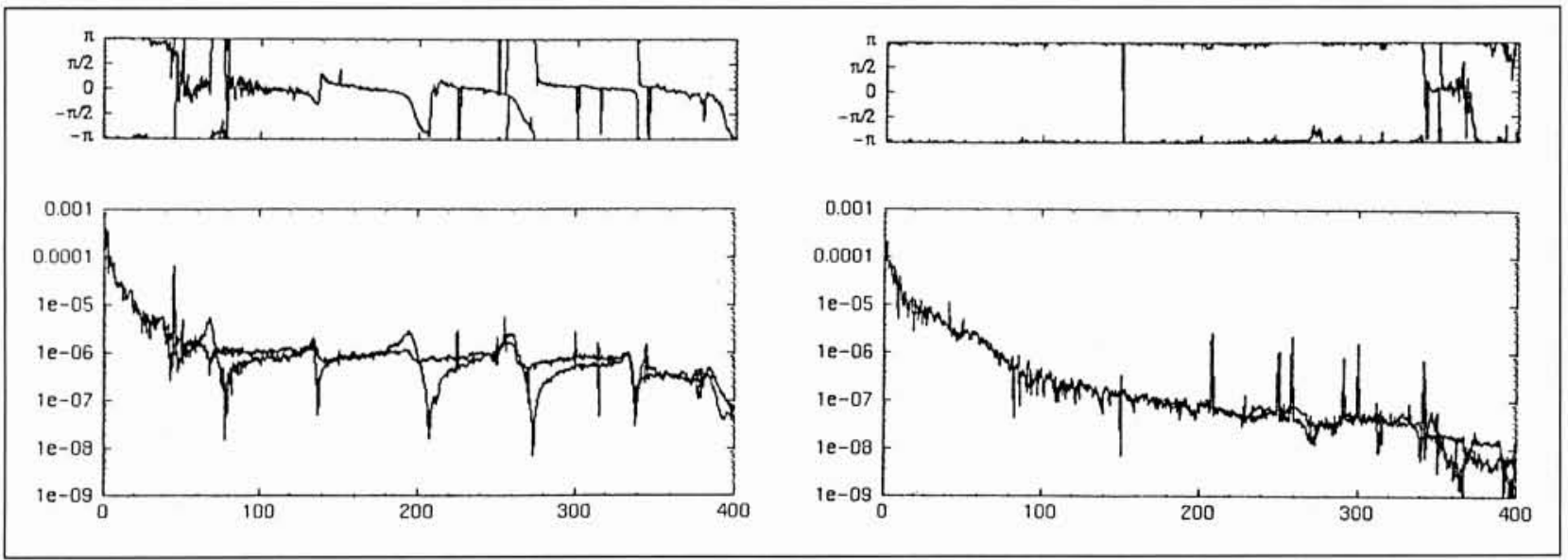

8. Spectres des sources pour le modèle à 2 sources de débit à droite pour un essai peu cavitant $(\sigma=5,4)$ et à gauche pour un essai cavitant $(\sigma=2)$. 
nues du modèle. Elle permet au moins de vérifier rapidement qu'aucune grosse erreur n'a été commise.

Les trois façons suivantes sont basées sur les deux modes de fonctionnement de CIRCUS : calcul prédictif (ou direct) et calcul diagnostic (ou inverse). Elles nécessitent la mise au point d'un modèle acoustique de la veine d'essais.

- Un calcul direct CIRCUS fournit le vecteur d'état (pi, qi) qui est comparé au vecteur mesuré au même point.

- Un calcul inverse CIRCUS fournit une valeur de la source qui est comparée à la source identifiée par intensimétrie.

- Une variante très intéressante de la méthode précédente consiste à considérer que les conditions limites sont inconnues et à comparer les coefficients de réflexion calculés par CIRCUS avec ceux obtenus par intensimétrie.

Après une brève présentation du modèle de la veine d'essai, nous allons détailler les 3 dernières méthodes, car elles permettent de juger la validité des 3 modèles de vanne proposés.

\subsection{Le modèle CIRCUS de la veine d'essai de vanne de la boucle EPOCA}

Devant la complexité de la boucle EPOCA, il parait illusoire (et probablement inutile) de chercher à modéliser la boucle complète. La solution retenue est probablement celle qui apporte le meilleur rapport qualité/difficulté : la modélisation de la veine d'essai vanne limitée par les 2 dilatoflex. Les avantages sont évidents :

- les mesures sont en quantité suffisante,

- la veine d'essai est composée uniquement de tuyaux droits, sans singularité,

- le couplage fluide/structure est a priori négligeable,

- la veine d'essai est bien découplée mécaniquement du reste du circuit et de la structure qui la supporte.

La difficulté principale réside dans la modélisation correcte des conditions limites. Les dilatoflex ne sont pas disponibles dans la base de données CIRCUS; il est donc nécessaire de fournir leurs caractéristiques - qui se résument ici à de "simples " coefficients de réflexion. Ces deux coefficients de réflexion sont déterminés par intensimétrie hydroacoustique (à l'aide du logiciel LADY). L'allure des coefficients de réflexion varie avec le dilatoflex considéré et le point d'essai $\left({ }^{8}\right)$. Il n'y a donc plus un modèle hydroacoustique de la veine d'essai, mais autant de modèles que de points d'essai, ce qui complique considérablement les validations.

\section{- 5.3 Le calcul direct CIRCUS}

Un calcul direct CIRCUS a été réalisé à partir du modèle de la veine, de la matrice $[M]$ et de la source $(\Delta p s, \Delta q s)$ pour chacun des 3 modèles hydroacoustiques de la vanne cavitante. Il permet de calculer en tous les points le vecteur d'état ( $p i, q i)$ qu'il suffit de comparer au vecteur mesuré au même point.

Les résultats obtenus sont présentés sur la figure 10 pour un essai cavitant $\sigma=2$ représentatif de l'étude. Les observations sont comparables pour tous les essais analysés mais ne sont pas présentées dans cet article.

\section{Conclusion}

L'examen des résultats montre que la meilleure concordance est obtenue pour le modèle à 2 sources de débit, aussi bien au niveau des modes, qu'entre ceux-ci et en basse fréquence.

\subsection{Le calcul inverse CIRCUS}

Un calcul inverse CIRCUS a été réalisé à partir du modèle de la veine, de la matrice de transfert $[M]$ et des mesures de pression fluctuante issues de capteurs en champ lointain, pour les 3 modèles hydroacoustiques de la vanne cavitante. Le code CIRCUS fournit une valeur de la source qu'il suffit de comparer à la source identifiée par intensimétrie.

(8) Nous pensons que ces différences sont dues à la différence de pression statique entre l'amont et l'aval d'une part, et entre les différents points d'essais d'autre part.

(9) le modèle CIRCUS de la veine pris en compte est identique pour les 3 modèles de vanne, notamment au niveau des conditions limites. Les seules différences se situent au niveau de la représentation de la vanne.
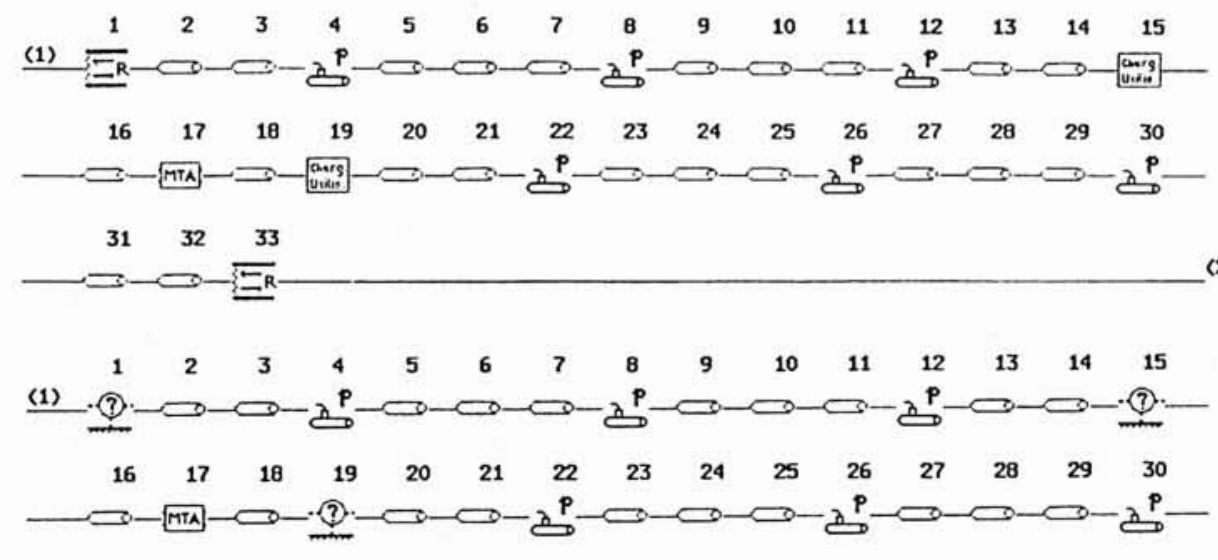

$\begin{array}{lll}31 & 32 & 33\end{array}$

9. Schéma du modèle hydroacoustique CIRCUS de la veine d'essai pour un calcul prédictif (direct) et pour un calcul diagnostic (inverse) dans le cas d'un modèle à 2 sources de débit $\left({ }^{9}\right)$. 


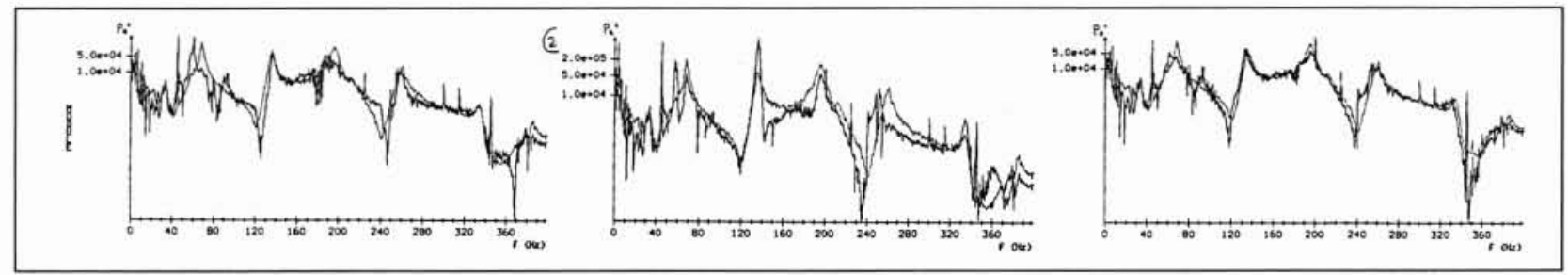

10. Comparaison d'un autospectre calculé avec CIRCUS direct et d'un autospectre mesuré. Modèle "standard " (à gauche), modèle à 5 zones (au milieu) et modèle à 2 sources de débit (à droite).

Les spectres des sources amont et aval sont calculés avec la matrice de transfert approchée égale à la matrice de transfert d'un tuyau droit équivalent. Les résultats sont présentés sur la figure 11 pour le modèle à 2 sources de débit.

Pour l'essai cavitant, les spectres obtenus sont quasiment identiques en module avec LADY et avec CIRCUS.

Pour l'essai non cavitant, on note une parfaite concordance entre les résultats de LADY et de CIRCUS.

Le code permet, ensuite, de calculer en tous les points le vecteur d'état $(p i, q i)$ à partir de la matrice de transfert $[M]$ et de la source associée identifiée par CIRCUS. Les autospectres obtenus sont comparés aux valeurs mesurées pour un capteur n'ayant pas servi à l'identification. Un exemple de cette comparaison est proposé dans la figure 12.

\subsection{Le calcul inverse CIRCUS avec conditions limites inconnues}

Une variante très intéressante de la méthode précédente consiste à considérer que les conditions limites sont inconnues. Le calcul inverse fournit donc les sources ainsi que les deux conditions limites, et en plus de comparer les sources, il est possible de comparer les coefficients de réflexion avec ceux obtenus par intensimétrie. Les coefficients de réflexion obtenus par le calcul inverse CIRCUS sont comparables à ceux obtenus par intensimétrie (fig. 13). A l'aval, la légère différence - qui correspond à une dizaine de centimètres est probablement due au fait que la réflexion ne se produit pas exactement au centre du dilatoflex.
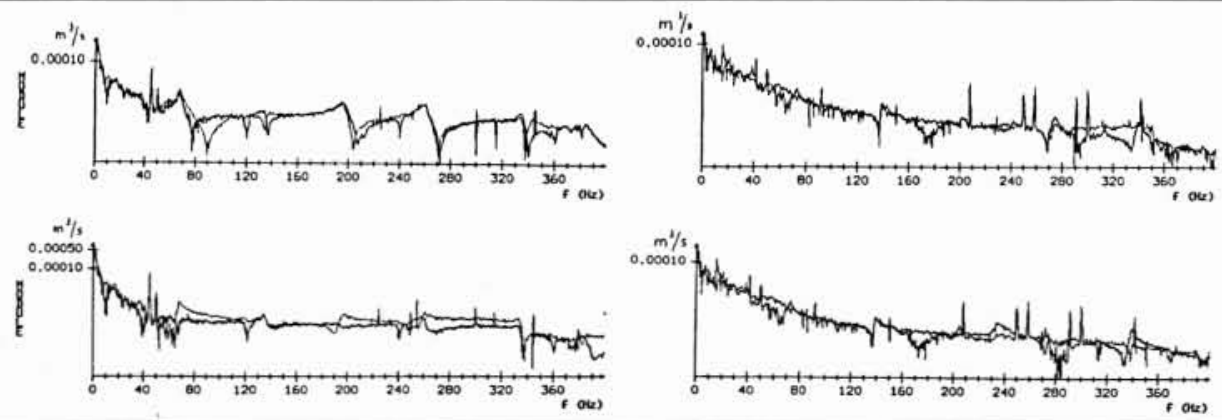

11. Comparaison des spectres des sources obtenus par CIRCUS et par intensimétrie à gauche pour un essai cavitant $(\sigma=2)$ et à droite pour un essai non cavitant $(\sigma=5,4)$.

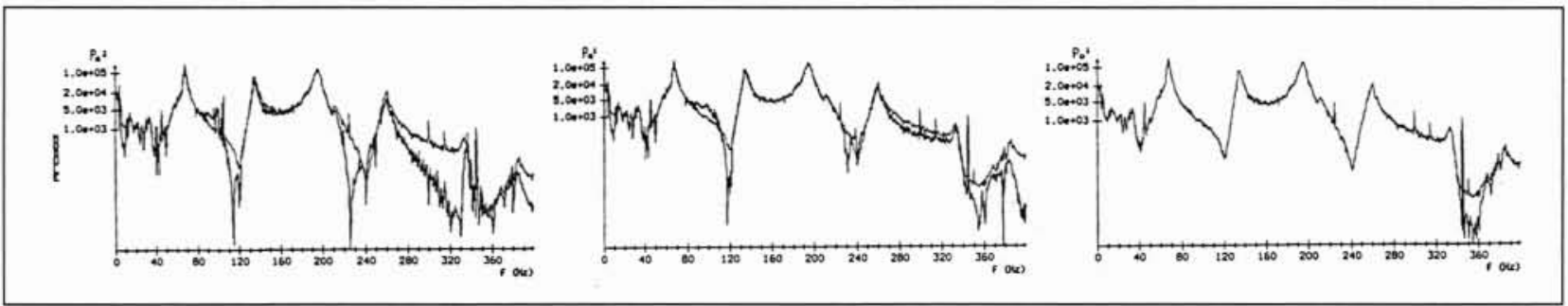

12. Comparaison d'un autospectre calculé avec CIRCUS inverse et d'un autospectre mesuré. Modèle «standard " (à gauche), modèle à 5 zones (au milieu) et modèle à 2 sources de débit (à droite).
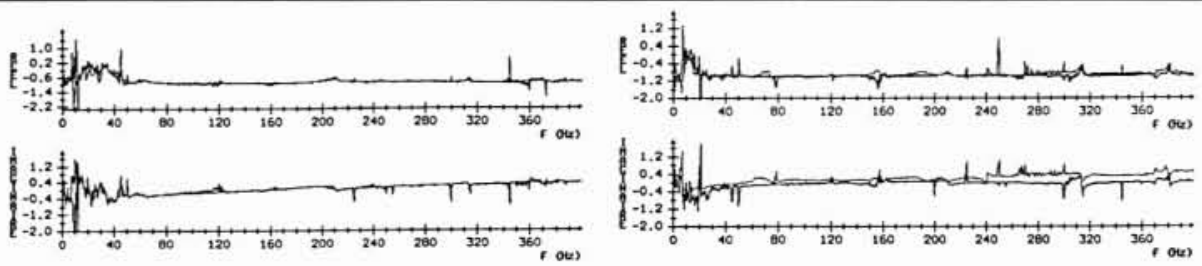

13. Comparaison des spectres des coefficients de réflexion obtenus par CIRCUS et par intensimétrie à gauche pour un essai cavitant $(\sigma=2)$ et à droite pour un essai non cavitant $(\sigma=5,4)$. 


\subsection{La conclusion de la validation}

La démarche de validation proposée montre une bonne concordance entre les résultats LADY et CIRCUS et confirme qu'un modèle de type sources/matrice de transfert permet de décrire correctement le comportement hydroacoustique d'une vanne Papillon. Le modèle à deux sources de débit semble le plus efficace en régime cavitant.

\section{ILES CONCLUSIONS ET LES PERS- PECTIVES}

Les conclusions qualitatives de la première campagne sont confirmées :

- la transition hydroacoustique entre un régime non cavitant et un régime cavitant $(\sigma=3)$ ne suit pas l'apparition visuelle de la cavitation $(\sigma=5)$,

- la source hydroacoustique n'est pas située au centre de la vanne, mais en aval de celle-ci,

- en dehors de la zone cavitante, pour laquelle il est difficile de déterminer les paramètres de propagation, l'hypothèse d'une propagation en ondes planes est démontrée.

La mise en œuvre aisée de l'excitatrice de débit a permis de générer des perturbations hydroacoustiques très supérieure à celles générées par la vanne et de déterminer expérimentalement les paramètres d'un modèle hydroacoustique de type source/matrice de transfert pour tous les régimes étudiés.

Pour décrire le comportement hydroacoustique d'une vanne Papillon en régime cavitant, 3 modèles ont été testés : - le modèle «standard" à 3 zones homogènes donne de bons résultats,

- le modèle à 5 zones est sans doute le plus proche de la réalité, mais donne des résultats moins satisfaisants (à cause de la difficulté à déterminer avec précision les paramètres de propagation dans la zone cavitante),

- le modèle à 2 sources de débit donne d'excellents résultats, ne nécessite pas la connaissance précise des paramètres de propagation dans la zone cavitante et il est directement compatible avec le code CIRCUS.

La mise au point d'un modèle de la veine d'essai de la boucle EPOCA a permis de tester et de comparer les différents modèles à l'aide de CIRCUS et de montrer que la qualité des résultats est largement suffisante pour ne utilisation comme chargement dans un calcul prédictif CIRCUS.
C'est donc le modèle à 2 sources de débit qui a été retenu et qui sera implanté dans la base de données du code CIRCUS.

L'analyse exhaustive des mesures de la base de données expérimentale est en cours. Une adimensionnalisation partielle en fonction du taux de cavitation et du débit a été réalisée pour une ouverture. La matrice de transfert varie faiblement en fonction du taux de cavitation et du débit et peut être modélisée par la matrice d'un tuyau droit équivalent. Les sources varient fortement en fonction du taux de cavitation à partir de $\sigma=2,5$ mais très peu avec le débit.

Il reste à confirmer et à affiner ce travail pour les 2 autres ouvertures étudiées, puis à essayer de trouver l'influence de l'ouverture de la vanne.

Une campagne d'essais sur une vanne Papillon de diamètre différent est prévue, pour prouver que le modèle retenu est valide quel que soit le diamètre de la vanne et appréhender l'évolution du modèle en fonction du diamètre.

Il serait d'autre part intéressant de comparer les résultats expérimentaux à ceux obtenus à partir d'une modélisation numérique du comportement hydroacoustique d'une vanne en régime cavitant... dès qu'un code numérique permettra de l'effectuer.

\section{BIBLIOGRAPHIE}

[1] Jacob T., Trollat C. (1991). - Hydroacoustic caracteristics of centrifugal pumps. IAHR-WG-Milan.

[2] Villouvier V. - Détermination expérimentale de sources et de matrices de transfert acoustiques: méthodes et moyens d'essais. EDF/DER-HP-63/92.172.

[3] Lauro J.-F., Boyer A. (1993). - Hydroacoustique d'une pompe - caractérisation des transferts dans une pompe au moyen d'une excitatrice de tuyauterie. AIRH-WG1-Lausanne.

[4] Hassis H., Lauro J.-F. et al. (1996). - Noise caused by cavitating Butterfly and Monovar valves. ASME-SanDiego.

[5] Boyer A., LAUro J.-F. - Etude hydroacoustique d'une vanne Papillon en régime cavitant. Rapport de synthèse après la $1^{\text {ère }}$ série d'essais. EDF/DER-HP-41/95/028.

[6] Boyer A., LAuro J.-F. - Etude hydroacoustique d'une vanne Papillon en régime cavitant. Méthodes et Résultats. EDF/DER-HP-41/97/005. 